\title{
Linear relations among theta series of genera of ternary forms
}

\author{
by
}

\section{Y. Choie and Y. Chung (Pohang)}

1. Introduction. It is well known that the orthogonal complement of the subspace of cusp forms with respect to the Petersson inner product is generated by the Eisenstein series of weight $k \geq 5 / 2$. Moreover, in [5] it was shown that the orthogonal complement $E_{3 / 2}\left(4 D, \chi_{\ell}\right)$ of the space of cusp forms of weight $3 / 2$ with level $4 D, D$ a square free integer, is generated by some Eisenstein series, which were explicitly constructed. Here $\ell$ is a positive divisor of $D$.

In this paper we compute the dimension of the space spanned by the theta series of the genera of positive definite ternary forms of level $4 D$ and find linear relations among them; first we find all distinct genera of positive definite ternary forms of level $4 D, D$ square free, with character $\chi_{\ell}$ and find a maximal independent set of the space spanned by the genus theta series. Secondly, by checking the values of the genus theta series at all cusps of $\Gamma_{0}(4 D)$ explicitly, linear relations among them are found. As a result we show that the Eisenstein space $E_{3 / 2}\left(4 P, \chi_{\ell}\right)$ of prime level $P$ is spanned by the theta series of the genera of positive definite ternary forms.

2. Number of genera of positive definite ternary forms. In this section we find all genera of ternary forms of level $4 D, D$ square free, and character $\chi_{\ell}$ using the local behavior of ternary forms. For more detailed results we refer to $[3]$.

Two integral quadratic forms are said to be semi-equivalent if they are equivalent over the $p$-adic integers for all primes $p$, and are equivalent over the real numbers (see [3] for more details). Semi-equivalent forms are said to be in the same genus of forms; equivalent forms are semi-equivalent, so we say classes of forms belong to a genus. To count the number of genera

2000 Mathematics Subject Classification: Primary 11F11, 11E20.

Key words and phrases: ternary forms, linear relations, theta series.

This work was partially supported by KOSEF R01-2003-00011596-0 and KRF 2003070-C00001. 
of positive definite ternary forms having level $4 D$ and character $\chi_{\ell}$ we first need the following lemmas. Throughout this paper, $D$ denotes a square free integer and $\ell$ is its divisor.

Lemma 2.1. Let $p$ be an odd prime. Let $f$ be a ternary form of level $4 D$ and character $\chi_{\ell}, \ell \mid D$. Let $\alpha, \beta$ and $\gamma$ be $p$-adic units and $\epsilon$ be a nonsquare p-adic unit.

(1) If $p \mid D$ and $p \mid \ell$, then $f$ is equivalent to $\alpha x^{2}+\beta y^{2}+\gamma p z^{2}$ over $\mathbb{Z}_{p}$. Moreover there are two equivalence classes of this type with discriminant $p$ or $\epsilon p$ over $\mathbb{Z}_{p}$.

(2) If $p \mid D$ and $p \nmid \ell$, then $f$ is equivalent to $\alpha x^{2}+\beta p y^{2}+\gamma p z^{2}$ over $\mathbb{Z}_{p}$.

(3) If $p \nmid D$, then $f$ is equivalent to $\alpha x^{2}+\beta y^{2}+\gamma z^{2}$. Moreover, there are two equivalence classes of this type with discriminant $p^{2}$ or $\epsilon p^{2}$ over $\mathbb{Z}_{p}$.

(4) There is a unique equivalence class of unimodular forms over $\mathbb{Z}_{p}$ with discriminant $\epsilon$ or 1 .

(5) For all the above forms, their equivalence classes are determined by the discriminant of their unimodular part.

Proof. It is clear that $x^{2}+y^{2}+p z^{2}$ and $x^{2}+\epsilon y^{2}+\epsilon p z^{2}$ are the only inequivalent forms having discriminant $p$. Also, $x^{2}+\epsilon y^{2}+p z^{2}$ is the only form (up to equivalence) having discriminant $\epsilon p$. The remaining assertions are similar by looking at the discriminant, so we omit the detailed proof.

Next we consider ternary forms with level $4 D$ over $\mathbb{Z}_{2}$.

Lemma 2.2. Let $f$ be a ternary form with level $4 D$ and character $\chi_{\ell}$. Let $\alpha, \beta, \gamma, \alpha_{i}, i=1,2,3$, and $\beta_{1}$ be units in $\mathbb{Z}_{2}$. Then:

(1) $f$ is equivalent to one of the forms $\alpha_{2} x^{2}+y z, \alpha x^{2}+\beta y^{2}+\gamma z^{2} \cong$ $\alpha_{1} x^{2}+2\left(\beta_{1} y^{2}+\beta_{1} z^{2}+y z\right)$ or $\alpha_{3} x^{2}+4 y z$ over $\mathbb{Z}_{2}$. Here $\beta_{1} \in\{0,1\}$. Furthermore, even if $\alpha_{i}, i=1,2,3$, may be distinct, their values modulo 4 are all the same.

(2) The number of equivalence classes of the type $\alpha x^{2}+\beta y^{2}+\gamma z^{2}$ is 2 .

(3) The number of equivalence classes of the type $\alpha x^{2}+y z$ is 1 .

(4) There is one-to-one correspondence between the forms of the type $\alpha x^{2}+y z$ and those of the type $\alpha x^{2}+4 y z$.

Proof. The results can be obtained by direct computation and we omit the detailed proof.

The following theorem is a necessary and sufficient condition for the existence of a form over $\mathbb{Q}$ with given local conditions.

Theorem 2.3 ([4, p. 203]). Suppose an n-ary quadratic space $V_{(p)}$ is given over $\mathbb{Q}_{p}$. In order that there exists an $n$-ary space $V$ over $\mathbb{Q}$ such that $V_{(p)} \equiv V_{p}$ for all primes $p$, it is necessary and sufficient that 
(1) there is a $d_{0} \in \mathbb{Q}$ with $d V_{(p)}=d_{0}$ for all $p$,

(2) $S_{p} V_{(p)}=1$ for almost all $p$,

(3) $\prod_{p} S_{p} V_{(p)}=1$.

Here, $S_{p} V_{(p)}$ denotes the Hasse symbol defined as follows. If $V$ is a binary quadratic space over $\mathbb{Q}$, then a quadratic form $f$ on $V$ can be written as $f(x, y)=a_{1} x^{2}+a_{2} y^{2}$ with $a_{1}, a_{2} \in \mathbb{Q}$. The Hasse symbol $S_{p} V=\left(a_{1}, a_{2}\right)$ at $p$ is given by

$$
\left(a_{1}, a_{2}\right):= \begin{cases}1 & \text { if } a_{1} x^{2}+a_{2} y^{2}=1 \text { is solvable in } \mathbb{Q}_{p}, \\ -1 & \text { otherwise }\end{cases}
$$

The Hasse symbol on an $n$-ary quadratic space $V$ is defined by

$$
S_{p} f=\prod_{1 \leq i \leq j \leq n}\left(a_{i}, a_{j}\right) \quad \text { for } f=\sum_{i=1}^{n} a_{i} x_{i}^{2} \in V .
$$

The following theorem gives the number of genera of positive definite ternary forms having level $4 D$ and character $\chi_{\ell}$. A more general result was stated in [3]. Here, we deal only with the case of a square free level $D$.

THEOREM 2.4. Let $g_{\ell}(4 D)$ be the number of genera of positive definite ternary forms having level $4 D, D$ square free, with character $\chi_{\ell}, \ell \mid D$. Then

$$
g_{\ell}(4 D)=2^{t(D)+1} .
$$

Here, $t(D)$ denotes the number of distinct prime factors of $D$.

Proof. Let $f$ be such a ternary form. We consider the following three cases:

(1) Suppose that $f$ is equivalent to $\alpha x^{2}+y z$ over $\mathbb{Z}_{2}$. Lemma 2.2 implies that this is the only equivalence class over $\mathbb{Z}_{2}$. Hence the Hasse symbol of $f$ at the prime 2 takes exactly one value among 1 or -1 and the Hasse symbol of $f$ at an odd prime $p, p \mid D$, may be taken both 1 and -1 . So, by Theorem 2.3(3), the number of genera of the above type is $2^{t(D)-1}$.

(2) Suppose that $f$ is equivalent to $\alpha x^{2}+\beta y^{2}+\gamma z^{2}$ over $\mathbb{Z}_{2}$. Lemma 2.2 shows that there are two distinct equivalence classes over $\mathbb{Z}_{2}$. Hence there are two possible values of the Hasse symbol of $f$ at the prime 2, that is, 1 or -1 . The Hasse symbol of $f$ at an odd prime $p, p \mid D$, can be 1 or -1 . Theorem 2.3 implies that in fact the number of genera of the above type is $2^{t(D)}$.

(3) If $f$ is equivalent to $\alpha x^{2}+4 x y$ over $\mathbb{Z}_{2}$, then by the same argument as in (2), we conclude that the number of genera of the above type is also $2^{t(D)-1}$.

Therefore, by summing all three cases we get the result. 
3. Theta series of genera of ternary forms. In this section we study theta series of genera of positive definite integral ternary forms and find their values at each cusp.

Let $f\left(x_{1}, x_{2}, x_{3}\right)$ be a positive definite quadratic form with integral coefficients, and $A_{f}=\left(\partial^{2} f / \partial x_{s} \partial x_{t}\right)$. Put

$$
\begin{gathered}
\theta_{f}(z)=\sum_{m \in \mathbb{Z}^{3}} \exp \left(z m A_{f} m^{t} / 2\right), \quad \exp (z):=e^{2 \pi i z}, \quad z \in \mathcal{H}, \\
0(f)=\#\left\{S \in M_{3 \times 3}(\mathbb{Z}) \mid S A_{f} S^{t}=A_{f}\right\} \\
\theta(\operatorname{gen} f, z)=\left(\sum_{f_{i}} \frac{1}{0\left(f_{i}\right)}\right)^{-1} \sum_{f_{i}} \frac{\theta_{f_{i}}(z)}{0\left(f_{i}\right)},
\end{gathered}
$$

where the $f_{i}$ run over a complete set of representatives of the equivalence classes in the genus of $f \cdot \theta($ gen $f, z)$ is called a theta series of genera of ternary forms or a genus theta series of ternary forms.

Then from the results in [6] and [7] we have the following theorem:

THEOREM 3.1. Let $f$ be a positive definite integral ternary form with level $N, N \equiv 0(\bmod 4)$, and $\chi=\left(\frac{2 \operatorname{det}\left(A_{f}\right)}{*}\right)$. Then:

(1) $\theta_{f}(z)$ belongs to $M_{3 / 2}(N, \chi)$,

(2) $\theta$ (gen $f, z)$ belongs to $E_{3 / 2}(N, \chi)$.

By evaluating $2^{t(D)+1}$ theta series of genera of ternary forms at all cusps, we find a maximal independent set of the space spanned by those. Moreover, linear relations among those genus theta series are listed; first note that the value of $\theta(\operatorname{gen} f, z)$ at each cusp is the same as that of $\theta_{f}(z)$. So we only need to compute the values of $\theta_{f}(z)$ at each cusp. Let $S(4 D)$ be a complete set of representatives of equivalence classes of cusps of $\Gamma_{0}(4 D)$. In fact, we can choose $S(4 D)=\{1 / t|t| 4 D\}$, and so $|S(4 D)|=3 \cdot 2^{t(D)}$.

The following theorem concerns the values of $\theta_{f}(z)$ at each cusp:

TheOREM $3.2([1])$. We have

$$
\lim _{z \rightarrow i \infty} \theta_{f}(z)=1
$$

The value of $\theta_{f}$ at each cusp $a / c \in S(4 D), a / c \neq \infty$, can be computed as

$$
V\left(\theta_{f}, a / c\right)=(-i)^{3 / 2}\left(\operatorname{det} A_{f}\right)^{-1 / 2} c^{-3 / 2} G_{A_{f}}(a, c),
$$

where $G_{A_{f}}(a, c)$ is the Gauss sum defined by

$$
G_{A_{f}}(a, c):=\sum_{x(\bmod c)} \exp \left(a x A_{f} x^{t} / 2 c\right) .
$$

The next proposition gives the values of Gaussian sums: 
Proposition 3.3 ([1]). (1) If $\left(c, c^{\prime}\right)=1$, then

$$
G_{A}\left(a, c c^{\prime}\right)=G_{A}\left(a c, c^{\prime}\right) G_{A}\left(a c^{\prime}, c\right) .
$$

(2) For each odd prime $p$, there exists an invertible matrix $S$ over the ring $\mathbb{Z}_{p}$ of $p$-adic integers such that

$$
S A S^{t}=\operatorname{diag}\left(\alpha_{1} p^{\beta_{1}}, \ldots, \alpha_{k} p^{\beta_{k}}\right),
$$

where $\alpha_{i}$, det $S \in \mathbb{Z}_{p}^{*}$ and $0 \leq \beta_{1} \leq \cdots \leq \beta_{k}$ are rational integers. Then

$$
\begin{aligned}
G_{A}\left(a, p^{m}\right) & =\sum_{x\left(\bmod p^{m}\right)} \exp \left(a x A x^{t} / 2 p^{m}\right) \\
& =p^{m l_{m}} \prod_{\beta_{i}<m} \frac{a \alpha_{i}^{\prime}}{p^{m-\beta_{i}}} \varepsilon_{p^{m-\beta_{i}}} p^{\left(m+\beta_{i}\right) / 2},
\end{aligned}
$$

where $\alpha_{i}^{\prime} \equiv 2^{-1} \alpha_{i}\left(\bmod p^{m-\beta_{i}}\right)$ and $\varepsilon_{d}=1$ or $i$ according as $d \equiv 1$ or $3(\bmod 4)$. Here, $l_{m}=\#\left\{\beta_{i} \mid \beta_{i} \geq m\right\}$.

(3) There exists an invertible matrix $S$ over the ring $\mathbb{Z}_{2}$ of 2-adic integers such that

$$
S A S^{t}=\bigoplus_{i=1}^{l} \alpha_{i} 2^{s_{i}} \oplus \bigoplus_{j=1}^{l_{1}} \beta_{j} 2^{t_{j}}\left(\begin{array}{ll}
0 & 1 \\
1 & 0
\end{array}\right) \oplus \bigoplus_{s=1}^{l_{2}} \gamma_{s} 2^{u_{s}}\left(\begin{array}{ll}
2 & 1 \\
1 & 2
\end{array}\right),
$$

where $\alpha_{i}, \beta_{j}, \gamma_{s} \in \mathbb{Z}_{2}^{*}$, and $s_{i} \geq 1, t_{j}, u_{s} \geq 0$ are rational integers. Here, $l_{m}=\#\left\{s_{i} \mid s_{i} \geq m+1\right\}+2 \#\left\{t_{j} \mid t_{j} \geq m\right\}+2 \#\left\{u_{s} \mid u_{s} \geq m\right\}$. Then

$$
\begin{aligned}
G_{A}\left(a, 2^{m}\right)= & 2^{m l_{m}} \prod_{s_{i}<m+1} G_{1, m+1-s_{i}}\left(a \alpha_{i}, 2^{m}\right) \\
& \times \prod_{t_{j}<m} 2^{m+t_{j}} \prod_{u_{s}<m}(-1)^{m-u_{s}} 2^{m+u_{s}},
\end{aligned}
$$

where

$$
G_{1, t}\left(a \alpha_{i}, 2^{m}\right)= \begin{cases}0 & \text { if } t=1, \\ \left(1+i^{a \alpha_{i}}\right) 2^{m-t / 2} & \text { if } t \text { is even, } \\ 2^{m-(t-1) / 2} e^{\pi i a \alpha_{i} / 4} & \text { if } t>1 \text { and odd } .\end{cases}
$$

Now we give the explicit value of $\theta_{f_{i}}$ at the cusp $a / c, V\left(\theta_{f_{i}}, a / c\right)$.

Proposition 3.4. Let $f_{i}=f_{i}(x, y, z)$ be a positive definite ternary form with level $4 D, D$ a square free odd positive integer. Let $\epsilon_{i j}$ be the discriminant of the unimodular part for $f_{i}$ over $\mathbb{Z}_{p_{j}}$, where $p_{j}$ is a prime such that $1 / p_{j} \in$ $S(4 D)$. 
(1) If $f_{i}$ is equivalent to $\alpha x^{2}+\beta y^{2}+\gamma p_{j} z^{2}$ over $\mathbb{Z}_{p_{j}}$, then

$$
V\left(\theta_{f_{i}}, 1 / p_{j}\right)=(-i)^{3 / 2}\left(\operatorname{det} A_{f_{i}}\right)^{-1 / 2} p_{j}^{-3 / 2} \varepsilon_{p_{j}}^{2}\left(\frac{\epsilon_{i j}}{p_{j}}\right) p_{j}^{2} .
$$

(2) If $f_{i}$ is equivalent to $\alpha x^{2}+\beta p_{j} y^{2}+\gamma p_{j} z^{2}$ over $\mathbb{Z}_{p_{j}}$, then

$$
V\left(\theta_{f_{i}}, 1 / p_{j}\right)=(-i)^{3 / 2}\left(\operatorname{det} A_{f_{i}}\right)^{-1 / 2} p_{j}^{-3 / 2} \varepsilon_{p_{j}}\left(\frac{\epsilon_{i j}}{p_{j}}\right) p_{j}^{5 / 2} .
$$

(3) If $f_{i}$ is equivalent to $f:=\alpha^{\prime} x^{2}+y z$ over $\mathbb{Z}_{2}$, then

$$
V\left(\theta_{f_{i}}, 1 / 4\right)=(-i)^{3 / 2}\left(\operatorname{det} A_{f_{i}}\right)^{-1 / 2} 4^{-3 / 2}\left(1+i^{\alpha^{\prime}}\right) 2 \cdot 2^{2} .
$$

(4) If $f_{i}$ is equivalent to $g_{\delta_{i}}:=\alpha x^{2}+\beta y^{2}+\gamma z^{2} \cong \alpha^{\prime} x^{2}+2 \delta y^{2}+2 \delta z^{2}+2 y z$ over $\mathbb{Z}_{2}$, then

$$
V\left(\theta_{f_{i}}, 1 / 4\right)=(-i)^{3 / 2}\left(\operatorname{det} A_{f_{i}}\right)^{-1 / 2} 4^{-3 / 2}\left(1+i^{\alpha^{\prime}}\right) 2 \cdot(-1)^{\delta} 2^{3} .
$$

Here, $\delta_{0}=0, \delta_{1}=1$.

(5) If $f_{i}$ is equivalent to $h:=\alpha^{\prime} x^{2}+4 y z$ over $\mathbb{Z}_{2}$, then

$$
V\left(\theta_{f_{i}}, 1 / 4\right)=(-i)^{3 / 2}\left(\operatorname{det} A_{f_{i}}\right)^{-1 / 2} 4^{-3 / 2}\left(1+i^{\alpha^{\prime}}\right) 2 \cdot 2^{4} .
$$

(6) $V\left(\theta_{f_{i}}, 1 / 2 v\right)=0$ for all $v \mid D$, for every ternary $f_{i}$.

Proof. Use Example 4.2 in [1] and Proposition 3.3. We omit the detailed proof.

In general we have the following:

Proposition 3.5. Let $f_{i}=f_{i}(x, y, z)$ be a positive definite ternary form of level $4 D, D$ square free, and character $\chi_{l}$, and let $c$ be a divisor of $D$. Then the value of the theta series of $f_{i}$ at each cusp is the following:

(1) We have

$$
\begin{aligned}
V\left(\theta_{f_{i}}, 1 / c\right)= & (-i)^{3 / 2}\left(\operatorname{det} A_{f_{i}}\right)^{-1 / 2} c^{-3 / 2} \prod_{p_{j} \mid c} G_{A_{f_{i}}}\left(1 / p_{j}, p_{j}\right) \\
= & (-i)^{3 / 2}\left(\operatorname{det} A_{f_{i}}\right)^{-1 / 2} c^{-3 / 2} \\
& \times \prod_{p_{j}\left|c, p_{j}\right| l} \varepsilon_{p_{j}}^{2}\left(\frac{\epsilon_{i j}}{p_{j}}\right) p_{j}^{2} \prod_{p_{j} \mid c, p_{j} \nmid l} \varepsilon_{p_{j}}\left(\frac{\epsilon_{i j} c / p_{j}}{p_{j}}\right) p_{j}^{5 / 2} .
\end{aligned}
$$

(2) If $f_{i}$ is equivalent to $f=\alpha^{\prime} x^{2}+y z$ over $\mathbb{Z}_{2}$, then

$$
\begin{aligned}
V\left(\theta_{f_{i}}, 1 / 4 c\right)= & (-i)^{3 / 2}\left(\operatorname{det} A_{f_{i}}\right)^{-1 / 2}(4 c)^{-3 / 2}\left(1+i^{\alpha^{\prime}}\right) 2 \cdot 2^{2} \\
& \times \prod_{p_{j}\left|c, p_{j}\right| l} \varepsilon_{p_{j}}^{2}\left(\frac{\epsilon_{i j}}{p_{j}}\right) p_{j}^{2} \prod_{p_{j} \mid c, p_{j} \nmid l} \varepsilon_{p_{j}}\left(\frac{\epsilon_{i j} c / p_{j}}{p_{j}}\right) p_{j}^{5 / 2} .
\end{aligned}
$$


(3) If $f_{i}$ is equivalent to $g_{\delta_{j}}=\alpha x^{2}+\beta y^{2}+\gamma z^{2} \cong \alpha^{\prime} x^{2}+2 \delta y^{2}+2 \delta z^{2}+2 y z$ over $\mathbb{Z}_{2}$, then

$$
\begin{aligned}
V\left(\theta_{f_{i}}, 1 / 4 c\right)= & (-i)^{3 / 2}\left(\operatorname{det} A_{f_{i}}\right)^{-1 / 2}(4 c)^{-3 / 2}\left(1+i^{\alpha^{\prime}}\right) 2 \cdot(-1)^{\delta} 2^{3} \\
& \times \prod_{p_{j}\left|c, p_{j}\right| l} \varepsilon_{p_{j}}^{2}\left(\frac{\epsilon_{i j}}{p_{j}}\right) p_{j}^{2} \prod_{p_{j} \mid c, p_{j} \nmid l} \varepsilon_{p_{j}}\left(\frac{\epsilon_{i j} c / p_{j}}{p_{j}}\right) p_{j}^{5 / 2} .
\end{aligned}
$$

(4) If $f_{i}$ is equivalent to $h=\alpha^{\prime} x^{2}+4 y z$ over $\mathbb{Z}_{2}$, then

$$
\begin{aligned}
V\left(\theta_{f_{i}}, 1 / 4 c\right)= & (-i)^{3 / 2}\left(\operatorname{det} A_{f_{i}}\right)^{-1 / 2}(4 c)^{-3 / 2}\left(1+i^{\alpha^{\prime}}\right) 2 \cdot 2^{4} \\
& \times \prod_{p_{j}\left|c, p_{j}\right| l} \varepsilon_{p_{j}}^{2}\left(\frac{\epsilon_{i j}}{p_{j}}\right) p_{j}^{2} \prod_{p_{j} \mid c, p_{j} \nmid l} \varepsilon_{p_{j}}\left(\frac{\epsilon_{i j} c / p_{j}}{p_{j}}\right) p_{j}^{5 / 2} .
\end{aligned}
$$

Proof. Use Propositions 3.3 and 3.4. We omit the detailed proof.

REMARK 3.6. Fix a cusp $1 / c$ and let $f_{i}$ run through all genera of level $4 D$ with a fixed character. According to Proposition 3.5 we note that:

(1) The factor $\left(\operatorname{det} A_{f_{i}}\right)^{-1 / 2} \prod_{p_{j} \mid c}\left(\frac{\epsilon_{i j}}{p_{j}}\right)$ in $V\left(\theta_{f_{i}}, 1 / c\right)$ is the only factor depending on $f_{i}$.

(2) The factor $\left(\operatorname{det} A_{f_{i}}\right)^{-1 / 2}(-1)^{\delta} 2^{*} \prod_{p_{j} \mid c}\left(\frac{\epsilon_{i j}}{p_{j}}\right)$ in $V\left(\theta_{f_{i}}, 1 / 4 c\right)$ is the only factor depending on $f_{i}$. Here $2^{*}$ denotes the power of 2 in $V\left(\theta_{f_{i}}, 1 / 4 c\right)$ and $\delta$ is defined in Proposition 3.5.

4. Main theorem. In this section we state our main result. First we need the following lemma.

Lemma 4.1 ([2, p. 319]). Let $G$ be a group and $K$ be a field. Let $\chi_{1}, \ldots$ $\ldots, \chi_{n}$ be distinct characters of $G$ in $K$. Then they are linearly independent over $K$.

TheOREM 4.2. Let $D$ be an odd square free positive integer and let $\ell$ be a divisor of $D$.

(1) The number of linearly independent genus theta series $\theta(\operatorname{gen} f, z)$ for ternary quadratic forms $f$ with level $4 D$ and character $\chi_{\ell}$ is $3 \cdot 2^{t(D)-1}$, where $t(D)$ is the number of prime factors dividing $D$.

(2) Let $f_{i}, g_{i}$ and $h_{i}$ be the ternary forms with the same discriminant in the unimodular part over $\mathbb{Z}_{p}$, for a prime $p \mid D$, which are equivalent to the forms $f=\alpha^{\prime} x^{2}+y z, g_{\delta_{0}}=\alpha^{\prime} x^{2}+2 y z$ and $h=\alpha^{\prime} x^{2}+4 y z$ over $\mathbb{Z}_{2}$, respectively. Then there is a linear relation among their genus theta series:

$$
3 \theta\left(\operatorname{gen} g_{i}, z\right)=\theta\left(\operatorname{gen} f_{i}, z\right)+2 \theta\left(\operatorname{gen} h_{i}, z\right) .
$$


Proof. Note that by Theorem 2.4 there are $2^{t(D)+1}$ genera of positive definite ternary forms having level $4 D$ and character $\chi_{\ell}$, and the number of nonzero $V\left(\theta_{f_{i}}, 1 / c\right)$ is $|S(4 D)|-2^{t(D)}=2^{t(D)+1}$. We consider the $2^{t(D)+1} \times$ $2^{t(D)+1}$ matrix

$$
H=\left(\begin{array}{cc}
V\left(\theta_{f_{i}}, 1 / c_{j}\right) & V\left(\theta_{f_{i}}, 1 / 4 c_{j}\right) \\
\ldots & \ldots \\
V\left(\theta_{g_{i}}, 1 / c_{j}\right) & V\left(\theta_{g_{i}}, 1 / 4 c_{j}\right) \\
\ldots & \ldots \\
V\left(\theta_{h_{i}}, 1 / c_{j}\right) & V\left(\theta_{h_{i}}, 1 / 4 c_{j}\right) \\
\ldots & \ldots
\end{array}\right)
$$

where $f_{i}, g_{i}$, and $h_{i}$ run over all genera with level $4 D$, character $\chi_{\ell}$ and $f_{i} \cong f=\alpha^{\prime} x^{2}+y z, g_{i} \cong g_{\delta_{j}}=\alpha^{\prime} x^{2}+2 \delta\left(y^{2}+z^{2}\right)+2 y z, j=0,1$, and $h_{i} \cong h=\alpha^{\prime} x^{2}+4 y z$ over $\mathbb{Z}_{2}$. Here, $1 / c_{j}, 1 / 4 c_{j} \in S(4 D)$ with $c_{j} \mid D$.

Our aim is to show that there are $3 \cdot 2^{t(D)-1}$ linearly independent rows in $H$. We write the matrix $H$ in the following form:

$$
H=\left(\begin{array}{cc}
A_{f, c_{j}} & A_{f, 4 c_{j}} \\
A_{g_{\delta_{0}}, c_{j}} & A_{g_{\delta_{0}}, 4 c_{j}} \\
A_{g_{\delta_{1}}, c_{j}} & A_{g_{\delta_{1}}, 4 c_{j}} \\
A_{h, c_{j}} & A_{h, 4 c_{j}}
\end{array}\right),
$$

where $A_{f, c_{j}}$ denotes the values of $\theta_{f_{i}}$ at the cusp $1 / c_{j}$ and $A_{f, 4 c_{j}}$ denotes the values of $\theta_{f_{i}}$ at the cusp $1 / 4 c_{j}$, where $f_{i}$ is equivalent to $f$. The other values are defined in a similar way. Here $\left\{c_{1}, \ldots, c_{2^{t(D)}}\right\}$ is the set of all divisors of $D$. By Proposition 3.5 and Remark 3.6, we can decompose the matrix $H$ as follows:

$$
\begin{aligned}
H= & \operatorname{diag}\left((-i)^{3 / 2}\left(\operatorname{det} A_{f_{i}}\right)^{-1 / 2}\right) \cdot H_{1} \\
& \cdot \operatorname{diag}\left(d_{j} \prod_{2<p_{j} \mid c_{j}, l \equiv 0\left(\bmod p_{j}\right)} \varepsilon_{p_{j}}^{2} p_{j}^{2} \prod_{2<p_{j} \mid c_{j}, l \neq 0\left(\bmod p_{j}\right)} \varepsilon_{p_{j}}\left(\frac{c / p_{j}}{p_{i}}\right) p_{j}^{5 / 2}\right),
\end{aligned}
$$

where

$$
d_{j}= \begin{cases}c_{j}^{-3 / 2} & \text { if } 1 \leq j \leq 2^{t(D)} \\ \left(4 c_{j}\right)^{-3 / 2} & \text { if } 2^{t(D)}<j \leq 2^{t(D)+1}\end{cases}
$$


and

$$
H_{1}:=\left(\begin{array}{clcc}
\prod_{p_{j} \mid c_{1}}\left(\frac{\epsilon_{1 j}}{p_{j}}\right) & \ldots \prod_{p_{j} \mid c_{1}}\left(\frac{\epsilon_{1 j}}{p_{j}}\right)\left(1+i^{c_{1} \alpha^{\prime}}\right) 2 \cdot 2^{2} & \ldots \\
\vdots & \vdots & \vdots \\
\prod_{p_{j} \mid c_{1}}\left(\frac{\epsilon_{2^{t(D)-1}+1, j}}{p_{j}}\right) & \ldots \prod_{p_{j} \mid c_{1}}\left(\frac{\epsilon_{2^{t(D)-1}+1, j}}{p_{j}}\right)\left(1+i^{c_{1} \alpha^{\prime}}\right) 2 \cdot 2^{3} & \ldots \\
\vdots & \vdots & \vdots & \vdots \\
\prod_{p_{j} \mid c_{1}}\left(\frac{\epsilon_{2^{t(D)}+1, j}}{p_{j}}\right) & \ldots \prod_{p_{j} \mid c_{1}}\left(\frac{\epsilon_{2^{t(D)}+1, j}}{p_{j}}\right)\left(1+i^{c_{1} \alpha^{\prime}}\right)(-1) 2 \cdot 2^{3} \ldots \\
\vdots & \vdots & \vdots & \vdots \\
\prod_{p_{j} \mid c_{1}}\left(\frac{\epsilon_{3 \cdot 2^{t(D)-1}+1, j}}{p_{j}}\right) & \ldots \prod_{p_{j} \mid c_{1}}\left(\frac{\epsilon_{3 \cdot 2^{t}(D)-1}+1, j}{p_{j}}\right)\left(1+i^{c_{1} \alpha^{\prime}}\right) 2 \cdot 2^{4} & \ldots
\end{array}\right) .
$$

Further note that

$$
H_{1}=H_{2} \cdot \operatorname{diag}\left(1,1, \ldots, 1,2^{3}\left(1+i^{c_{1} \alpha^{\prime}}\right), \ldots, 2^{3}\left(1+i^{c_{t(D)} \alpha^{\prime}}\right)\right)
$$

where

$$
\begin{aligned}
& H_{2}:=\left(\begin{array}{clcc}
\prod_{p_{j} \mid c_{1}}\left(\frac{\epsilon_{1 j}}{p_{j}}\right) & \ldots & \prod_{p_{j} \mid c_{1}}\left(\frac{\epsilon_{1 j}}{p_{j}}\right) & \ldots \\
\vdots & \vdots & \vdots & \vdots \\
\prod_{p_{j} \mid c_{1}}\left(\frac{\epsilon_{2^{t}(D)-1}+1, j}{p_{j}}\right) & \ldots & \prod_{p_{j} \mid c_{1}}\left(\frac{\epsilon_{2^{t(D)-1}+1, j}}{p_{j}}\right) 2 & \ldots \\
\vdots & \vdots & \vdots & \vdots \\
\prod_{p_{j} \mid c_{1}}\left(\frac{\epsilon_{2^{t(D)}+1, j}}{p_{j}}\right) & \ldots & \prod_{p_{j} \mid c_{1}}\left(\frac{\epsilon_{2^{t(D)}+1, j}}{p_{j}}\right)(-1) 2 & \ldots \\
\vdots & \vdots & \vdots & \vdots \\
\prod_{p_{j} \mid c_{1}}\left(\frac{\epsilon_{3 \cdot 2^{t(D)-1}+1, j}}{p_{j}}\right) & \ldots & \prod_{p_{j} \mid c_{1}}\left(\frac{\epsilon_{3 \cdot 2^{t(D)-1}+1, j}}{p_{j}}\right) 4 & \ldots \\
\vdots & \vdots & \vdots & \vdots
\end{array}\right) \\
& \cdot \operatorname{diag}\left(d_{j} \prod_{2<p_{j} \mid c_{j}, l \equiv 0\left(\bmod p_{j}\right)} \varepsilon_{p_{j}}^{2} p_{j}^{2} \prod_{2<p_{j} \mid c_{j}, l \neq 0\left(\bmod p_{j}\right)} \varepsilon_{p_{j}}\left(\frac{c / p_{j}}{p_{j}}\right) p_{j}^{5 / 2}\right) \\
& \cdot \operatorname{diag}\left(1,1, \ldots, 1,2^{3}\left(1+i^{c_{1} \alpha^{\prime}}\right), \ldots, 2^{3}\left(1+i^{c_{t(D)} \alpha^{\prime}}\right)\right) \text {. }
\end{aligned}
$$

Now it is enough to consider $H_{2}$ to find the linearly independent rows of $H$. First note that Hasse symbol $S_{p_{j}} f_{i}$, for each odd prime $p_{j}$, is determined by the square class $\left(\frac{\epsilon_{i j}}{p_{j}}\right)$. One also checks that $S_{2}(f)=S_{2}\left(g_{\delta_{0}}\right)=$ $S_{2}(h)$ and $S_{2}\left(g_{\delta_{1}}\right) \neq S_{2}\left(g_{\delta_{0}}\right)$. On the other hand, using Theorem 2.4, one finds that $\prod_{p \neq 2} S_{p}=S_{2}$. So, we may reduce the matrix $H_{2}$ to the following 
form:

$$
H_{2}=\left(\begin{array}{cc}
A & A \\
A & 2 A \\
A^{\prime} & -2 A^{\prime} \\
A & 4 A
\end{array}\right) .
$$

Here $A$ and $A^{\prime}$ are $2^{t(D)-1} \times 2^{t(D)}$-matrices. Since $\left(\begin{array}{c}A \\ A^{\prime}\end{array}\right)$ is a nonsingular matrix by Lemma 4.1, all rows of

$$
\left(\begin{array}{cc}
A & A \\
A & 2 A \\
A^{\prime} & -2 A^{\prime}
\end{array}\right)
$$

are linearly independent. Since $(A, 4 A)=3(A, 2 A)-2(A, A)$, we have $\left(\operatorname{det} A_{h_{i}}\right)^{1 / 2} \theta\left(\operatorname{gen} h_{i}, z\right)=3\left(\operatorname{det} A_{g_{i}}\right)^{1 / 2} \theta\left(\operatorname{gen} g_{i}, z\right)-2\left(\operatorname{det} A_{f_{i}}\right)^{1 / 2} \theta\left(\operatorname{gen} f_{i}, z\right)$. After dividing by the common factors of $\left(\operatorname{det} A_{h_{i}}\right)^{1 / 2},\left(\operatorname{det} A_{g_{i}}\right)^{1 / 2}$ and $\left(\operatorname{det} A_{f_{i}}\right)^{1 / 2}$, we have the stated linear relation between the genus theta series of ternary forms. The proof is complete.

COROLlary 4.3. The genus theta series of ternary forms with level $4 D$ and character $\chi_{\ell}$ generate the Eisenstein space $E_{3 / 2}\left(4 D, \chi_{\ell}\right)$ of weight $3 / 2$ and level $4 D$ with character $\chi_{\ell}$ if and only if $D$ is prime.

Proof. Note that $\operatorname{dim}\left(E_{3 / 2}\left(4 D, \chi_{\ell}\right)\right)=2^{t(D)+1}-1$ (see [5]). Since by Theorem 4.2 there are $2^{t(D)}+2^{t(D)-1}$ linearly independent genus theta series of ternary forms, $2^{t(D)}+2^{t(D)-1}=2^{t(D)+1}-1$ if and only if $D$ itself is an odd prime.

\section{Examples and theta identities}

ExAmple 5.1. Consider ternary forms with level $4 D=12$. In this case there are 4 different classes which belong to 4 different genera. More precisely, the table in [3] gives the following:

(1) Forms of level 12 and character $\chi_{1}=\mathrm{id}$ are $\left(\alpha_{i} \in \mathbb{Z}_{2}^{*}, i=1,2,3\right)$ :

(a) $f_{1}=x^{2}+y^{2}+3 z^{2}+x y$, equivalent to $f=\alpha_{1} x^{2}+y z$ over $\mathbb{Z}_{2}$,

(b) $g_{1}=x^{2}+3 y^{2}+3 z^{2}$, equivalent to $g_{\delta_{0}}=\alpha_{2} x^{2}+2 y z$ over $\mathbb{Z}_{2}$,

(c) $g_{2}=3 x^{2}+2\left(y^{2}+z^{2}+y z\right)$, equivalent to $g_{\delta_{1}}=3 x^{2}+2\left(y^{2}+z^{2}+y z\right)$ over $\mathbb{Z}_{2}$,

(d) $h_{1}=3 x^{2}+4 y^{2}+4 z^{2}+4 y z$, equivalent to $h=\alpha_{3} x^{2}+4 y z$ over $\mathbb{Z}_{2}$.

Theorem 4.2 implies the following linear relation among theta series:

$$
3 \sum_{x, y, z \in \mathbb{Z}} q^{x^{2}+3 y^{2}+3 z^{2}}=\sum_{x, y, z \in \mathbb{Z}} q^{x^{2}+y^{2}+3 z^{2}+x y}+2 \sum_{x, y, z \in \mathbb{Z}} q^{3 x^{2}+4 y^{2}+4 z^{2}+4 y z} .
$$


(2) Forms of level 12 and character $\chi_{3}$ are $\left(\alpha_{i} \in \mathbb{Z}_{2}^{*}, \quad i=1,2,3\right)$ :

(a) $f_{1}=x^{2}+y^{2}+z^{2}+x y$, equivalent to $f=\alpha_{1} x^{2}+y z$ over $\mathbb{Z}_{2}$,

(b) $g_{1}=x^{2}+y^{2}+3 z^{2}$, equivalent to $g_{\delta_{0}}=\alpha_{2} x^{2}+2 y z$ over $\mathbb{Z}_{2}$,

(c) $g_{2}=x^{2}+2 y^{2}+2 z^{2}+2 x y$, equivalent to $g_{\delta_{1}}=x^{2}+2\left(y^{2}+z^{2}+x y\right)$,

(d) $h_{1}=x^{2}+4 y^{2}+4 z^{2}+4 y z$, equivalent to $h=\alpha_{3} x^{2}+4 y z$ over $\mathbb{Z}_{2}$.

Theorem 4.2 implies the following linear relation among theta series:

$$
3 \sum_{x, y, z \in \mathbb{Z}} q^{x^{2}+y^{2}+3 z^{2}}=\sum_{x, y, z \in \mathbb{Z}} q^{x^{2}+y^{2}+z^{2}+x y}+2 \sum_{x, y, z \in \mathbb{Z}} q^{x^{2}+4 y^{2}+4 z^{2}+4 y z} .
$$

ExAmple 5.2. Consider ternary forms with level $4 D=28$. There are 5 different classes and only two of them belong to the same genus [3].

(1) Reduced ternary forms of level 28 and character $\chi=$ id are:

(a) $f_{1}=x^{2}+2 y^{2}+7 z^{2}+x y$, equivalent to $f=\alpha_{1} x^{2}+y z$ over $\mathbb{Z}_{2}$,

(b) $g_{1}=x^{2}+7 y^{2}+7 z^{2}, 2 x^{2}+4 y^{2}+7 z^{2}-2 x y$, equivalent to $g_{\delta_{0}}=$ $\alpha_{2} x^{2}+2 y z$ over $\mathbb{Z}_{2}$

(c) $g_{2}=3 x^{2}+5 y^{2}+5 z^{2}-4 y z-2 x z-2 x y$, equivalent to $g_{\delta_{1}}=\alpha_{3} x^{2}+$ $2\left(y^{2}+z^{2}+y z\right)$ over $\mathbb{Z}_{2}$

(d) $h_{1}=4 x^{2}+7 y^{2}+8 z^{2}-4 x z$, equivalent to $h=\alpha_{4} x^{2}+4 y z$ over $\mathbb{Z}_{2}$.

Theorem 4.2 implies the following linear relation among theta series:

$$
\begin{aligned}
\sum_{x, y, z \in \mathbb{Z}} q^{x^{2}+7 y^{2}+7 z^{2}} & +2 \sum_{x, y, z \in \mathbb{Z}} q^{2 x^{2}+4 y^{2}+7 z^{2}+2 x y} \\
& =\sum_{x, y, z \in \mathbb{Z}} q^{x^{2}+2 y^{2}+7 z^{2}+x y}+2 \sum_{x, y, z \in \mathbb{Z}} q^{4 x^{2}+4 y^{2}+4 z^{2}+4 y z} .
\end{aligned}
$$

(2) Reduced ternary forms of level 28 and character $\chi_{7}$ are:

(a) $f_{1}=x^{2}+y^{2}+2 z^{2}-x z$, equivalent to $f=\alpha_{1} x^{2}+y z$ over $\mathbb{Z}_{2}$,

(b) $g_{1}=x^{2}+y^{2}+7 z^{2}, x^{2}+2 y^{2}+4 z^{2}-2 y z$, equivalent to $g_{\delta_{0}}=\alpha_{2} x^{2}+2 y z$ over $\mathbb{Z}_{2}$,

(c) $g_{2}=2 x^{2}+2 y^{2}+3 z^{2}+2 y z+2 x z+2 x y$, equivalent to $g_{\delta_{1}}=\alpha_{3} x^{2}+$ $2\left(y^{2}+z^{2}+y z\right)$ over $\mathbb{Z}_{2}$

(d) $h_{1}=x^{2}+4 y^{2}+8 z^{2}-4 y z$, equivalent to $h=\alpha_{4} x^{2}+4 y z$ over $\mathbb{Z}_{2}$.

Theorem 4.2 implies the following linear relation among theta series:

$$
\begin{aligned}
\sum_{x, y, z \in \mathbb{Z}} q^{x^{2}+y^{2}+7 z^{2}}+ & 2 \sum_{x, y, z \in \mathbb{Z}} q^{x^{2}+2 y^{2}+4 z^{2}+2 y z} \\
& =\sum_{x, y, z \in \mathbb{Z}} q^{x^{2}+y^{2}+2 z^{2}+x z}+2 \sum_{x, y, z \in \mathbb{Z}} q^{x^{2}+4 y^{2}+8 z^{2}+4 y z} .
\end{aligned}
$$




\section{References}

[1] Y. Choie, D. Pei and X. Wang, The values of Eisenstein series at cusp points, preprint, 2002.

[2] S. Lang, Algebra, Addison-Wesley, 1993.

[3] L. Lehman, Levels of positive definite ternary quadratic forms, Math. Comp. 58 (1992), 399-417.

[4] O. T. O'Meara, Introduction to Quadratic Forms, Springer, New York, 1973.

[5] D. Pei, Eisenstein series of weight 3/2: I, II, Trans. Amer. Math. Soc. 274 (1982), 573-606 and 283 (1984), 589-603.

[6] R. Schulze-Pillot, Thetareihen positiv definiter quadratischer Formen, Invent. Math. 75 (1984), 283-299.

[7] G. Shimura, On modular forms of half integral weight, Ann. of Math. 97 (1973), 440-481.

Department of Mathematics

Pohang University of Science and Technology

Pohang, 790-784, South Korea

E-mail: yjc@postech.ac.kr ycc@postech.ac.kr 